\title{
Manipulation of polarizations for broadband terahertz waves emitted from laser plasma filaments
}

\author{
Zhelin Zhang, ${ }^{1,2}$ Yanping Chen, ${ }^{1,2}$, a) Sen Cui, ${ }^{1,2}$ Feng He, ${ }^{1,2}$ Min Chen, ${ }^{1,2}$ Zhen Zhang, ${ }^{1,2}$ Jin Yu, ${ }^{1}$ Liming \\ Chen, 1,2 Zhengming Sheng, 1, 2, 3, 4, 5, b) and Jie Zhang ${ }^{1,2}$ \\ ${ }^{1)}$ Key Laboratory for Laser Plasmas (Ministry of Education), School of Physics and Astronomy, \\ Shanghai Jiao Tong University, Shanghai 200240, China \\ ${ }^{2)}$ Collaborative Innovation Center of IFSA, Shanghai Jiao Tong University, Shanghai 200240, \\ China \\ 3) Department of Physics, SUPA, University of Strathclyde, Glasgow G4 ONG, \\ $U K$ \\ 4) Tsung-Dao Lee Institute, Shanghai 200240, China \\ ${ }^{5)}$ Cockcroft Institute, Sci-Tech Daresbury, Cheshire WA4 4AD, UK
}

(Dated: 17 August 2018)

Polarization control of broadband terahertz waves is essential for applications in many areas such as material sciences, medical and biological diagnostics, near-field communications and public securities. Conventional methods for polarization control are limited to narrow bandwidth and often with low efficiency. Here based upon theoretical and experimental studies, we demonstrate that the two-colour laser scheme in gas plasma can provide effective control of elliptically polarized terahertz waves, including their ellipticity, azimuthal angle, and chirality. This is achieved with a circularly-polarized laser at the fundamental frequency and its linearly polarized second harmonic, a controlled phase difference between these two laser components, as well as a suitable length of the laser plasma filament. A flexible control of their ellipticity and azimuthal angle is demonstrated with our theoretical model and systematic experiments. This offers a unique and flexible technique on the polarization control of broadband terahertz radiation suitable for wide applications.

Plasmas driven by two-colour femtosecond laser pulses in air are known to be able to emit broadband strong terahertz waves with reasonable efficiency suitable for many applications $^{1,2}$. For many of these applications, effective control of their polarization states is a key prerequisite. In material sciences, for instance, the polarization direction of a terahertz wave plays a pivotal role in optical excitations $^{3}$, spectroscopy diagnostics ${ }^{4-7}$, and terahertz information technologies ${ }^{8}$. Meanwhile, the ellipticity of the terahertz wave is an important parameter in birefringent materials imaging ${ }^{9}$ and its chirality is critical for studying chiral rotation of organic molecules and spin dynamics in solid materials ${ }^{10,11}$. Currently it is still a great challenge to realize flexible control of polarization states of the broadband terahertz pulses. Conventional methods, such as wire-grid polarizers and Fresnel-rhomb wave plates, have limited flexibility in the polarization modulation and are usually applicable to terahertz waves only with narrow spectral bandwidth ${ }^{12}$. Recently, it was demonstrated that linearly and circularly polarized terahertz pulses can emit from laser plasma sources with application of an external electrical field ${ }^{13-15}$. Moreover, terahertz pulses with linear and elliptical polarizations have been observed by two-colour coherent control of the motion of ionized electrons ${ }^{16-22}$. Qualitatively it was shown that the transformation of the polarization states always involved simultaneously both the azimuthal angle and ellipticity in these schemes, which restrict the

\footnotetext{
a) Electronic mail: yanping.chen@sjtu.edu.cn

b) Electronic mail: z.sheng@strath.ac.uk
}

flexibility of polarization modulation for practical applications.

Here, we introduce a new route to realize flexible control of polarization states for terahertz pulses produced in the two-colour laser scheme based upon theoretical and experimental investigations. We demonstrate that the polarized terahertz pulses with any desired azimuthal angle and ellipticity can be realized by controlling the relative phase between two-colour laser components and the length of a filament, respectively. Its polarization chirality can be easily tuned by changing the polarization of the pump lasers.

\section{RESULTS AND DISCUSSIONS}

Figure 1a sketches the experimental setup generating terahertz pulses with any polarization states in air plasmas excited by a two-colour laser pulse composed of a fundamental wave $(\mathrm{FW})$ and its second harmonic $(\mathrm{SH})$. Either for the terahertz pulses or the incident laser pulses, their polarization states are characterized by their ellipticity and azimuthal angle, which are the trajectories of the tip of electric field vectors projected on the $x ? y$ plane, denoted as $\left(\varepsilon^{\mathrm{THz}}, \theta^{\mathrm{THz}}\right),\left(\varepsilon^{\omega}, \theta^{\omega}\right),\left(\varepsilon^{2 \omega}, \theta^{2 \omega}\right)$ for the terahertz pulse and the incident laser FW and $\mathrm{SH}$, respectively. The initial relative phase between the FW and $\mathrm{SH}$ pulses $\left(\varphi_{d}\right)$ was controlled by laterally translating a fused silica wedge in an in-line phase compensator (IPC) consisting of an alpha-barium borate $(\alpha-$ $\mathrm{BBO}$ ) time plate, a pair of fused silica wedges and a dual wavelength plate $(\mathrm{DWP})^{16}$. The polarization of 

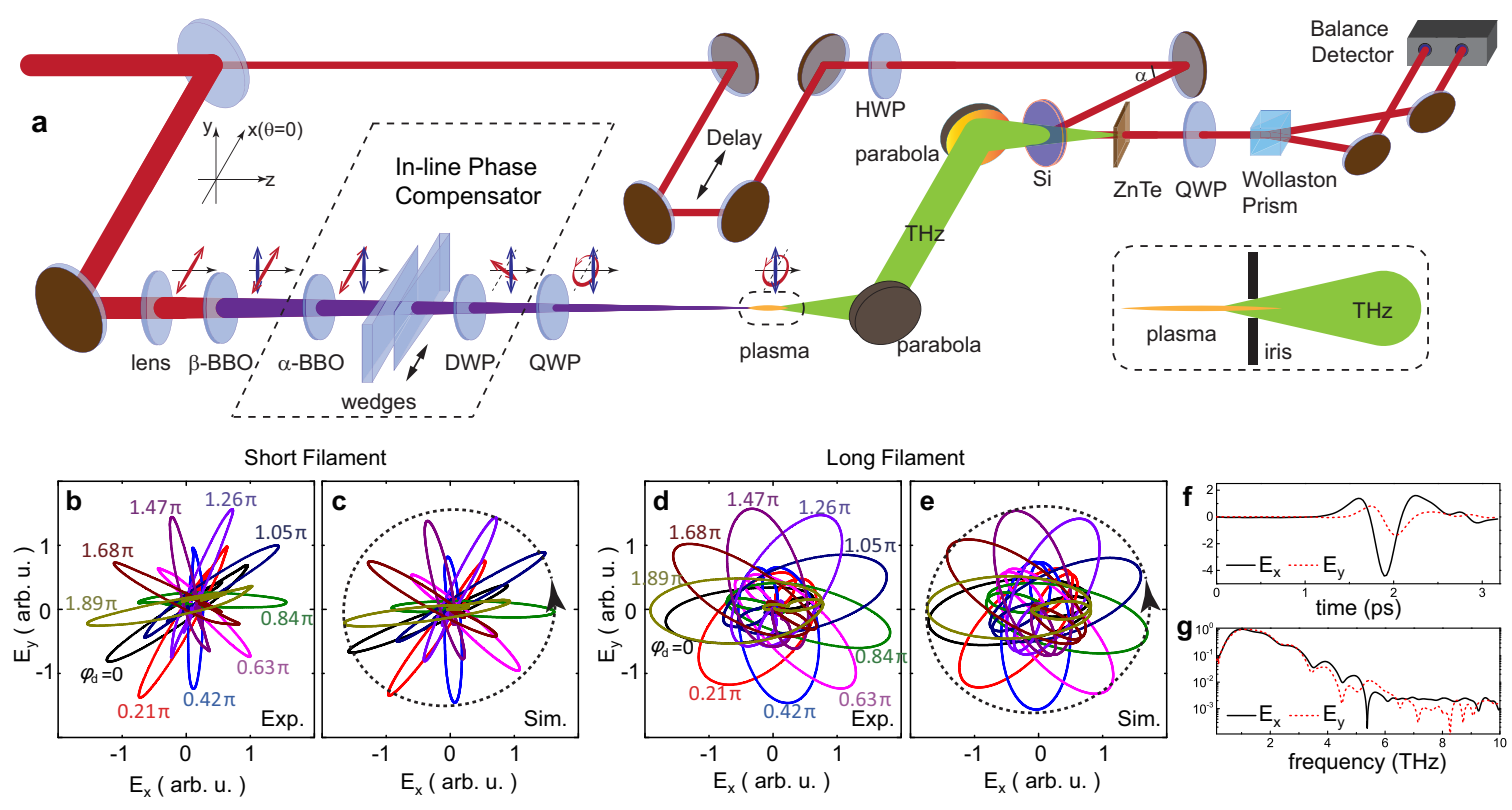

FIG. 1. Experimental demonstration of terahertz generation with arbitrary polarizations. (a) Schematic of the experimental setup. An $800 \mathrm{~nm}$ laser pulse of $40 \mathrm{fs}$ pulse duration and $4 \mathrm{~mJ}$ energy is focused by a plano-convex lens of 30 $\mathrm{cm}$ focal length to form a 23-mm-long laser filament in air. Before the laser pulse undergoes filamentation, it is intercepted by a $\beta$-BBO crystal of $0.2 \mathrm{~mm}$ thickness for $\mathrm{SH}$ generation and an IPC for relative phase change between the $\mathrm{FW}$ and $\mathrm{SH}$ pulses. Both the $\mathrm{FW}$ and $\mathrm{SH}$ pulses are linearly polarized after the IPC, where their polarization directions differs by 46 degrees $\left(\theta^{\omega} \approx-\pi / 4\right.$ and $\left.\theta^{2 \omega}=\pi / 2\right)$. Thereafter, the FW pulse was changed to a circularly polarized one by a quarter wave plate (QWP, $800 \mathrm{~nm}$ ) with its optical axis parallel to the polarization direction of the SH pulse. A probe beam, split from the $800 \mathrm{~nm}$ input laser, is synchronized to the generated terahertz pulse inside the ZnTe crystal for terahertz measurement. The inset illustrates modification of filament length by moving a metal iris of 2-mm-aperture. $\alpha=40^{\circ}$. (b) and (d) show the far-field polarization trajectories $\left[E_{x}(t), E_{y}(t)\right]$ of terahertz radiations obtained experimentally from a short filament and a long filament, respectively, when varying $\varphi_{d}$ with an interval of $0.21 \pi$. The corresponding simulations are shown in (c) for a short filament and (e) for a long filament, respectively. Polarization is defined from the point of view of the receiver. (f) and (g) are typical waveforms and spectra of measured elliptically polarized radiation from a 23-mm-long filament.

the far-field terahertz pulse was characterized by composing two orthogonally polarized electric field vectors of this terahertz pulse measured by an electro-optical sampling method with a 1-mm-thick ZnTe $<110>$ crystal in two orthogonal directions ${ }^{23,24}$ (See Methods for measurement for terahertz polarization). The polarization of the FW pulse $\left(\varepsilon^{\omega}, \theta^{\omega}\right)$ is controllable by managing the DWP and the QWP located after the IPC simultaneously. In our scheme, the $\mathrm{FW}$ is almost circularly polarized with $\varepsilon^{\omega}=0.95$ and $\theta^{\omega}=0$, while the polarization of the $\mathrm{SH}$ pulse remains to be linearly polarized with $\varepsilon^{2 \omega}=0$ and $\theta^{2 \omega}=\pi / 2$. As shown in the section 'Manipulation of terahertz polarization', these polarization states for the driving laser can provide the highest flexibility of terahertz polarization. The filament length $L_{\mathrm{fm}}$ for effective delivery of conical forward terahertz pulses can be modified by moving a metal iris along this filament since terahertz radiation from a laser filament is confined in a cone-shape and cannot transmit through metals ${ }^{25,26}$. With $\left(\varepsilon^{\omega}, \theta^{\omega}\right)$ and $\left(\varepsilon^{2 \omega}, \theta^{2 \omega}\right)$ given above together with variable $L_{\mathrm{fm}}, \varphi_{d}$, one can modulate flexibly the terahertz polarization state $\left(\varepsilon^{\mathrm{THz}}, \theta^{\mathrm{THz}}\right)$.

Dependence of terahertz wave polarization upon filament length and relative phase of two colour laser fields. For the given polarization states for the two-colour fields with the relative phase $\varphi_{d}$, an emitted terahertz pulse from the laser filament with the length $L_{\mathrm{fm}}$ is usually elliptically polarized with certain $\left(\varepsilon^{\mathrm{THz}}, \theta^{\mathrm{THz}}\right)$. When either $L_{\mathrm{fm}}$ or $\varphi_{d}$ changes, usually both $\varepsilon^{\mathrm{THz}}$ and $\theta^{\mathrm{THz}}$ are expected to change. For a fixed filament length of $L_{\mathrm{fm}}=4 \mathrm{~mm}$, as $\varphi_{d}$ changes from 0 to $2 \pi$, the measured far-field terahertz polarization states are illustrated in Fig. 1b. For each $\varphi_{d}$, the terahertz wave is elliptically polarized with a similar ellipticity about $\varepsilon^{\mathrm{THz}}=0.1$, and its azimuthal angle $\theta^{\mathrm{THz}}$ rotates anticlockwise with respect to the original point. In addition, the chirality of the terahertz polarization preserves lefthanded in the present case. On the contrast, when the length of the filament is around $L_{\mathrm{fm}}=23 \mathrm{~mm}$, the ellipticity of the terahertz wave increases to $\varepsilon^{\mathrm{THz}}=0.48$ while the characteristics of its chirality and azimuthal angle regarding $\varphi_{d}$ show the same behaviors as that obtained in a short filament (Fig. 1d). These observations suggest 

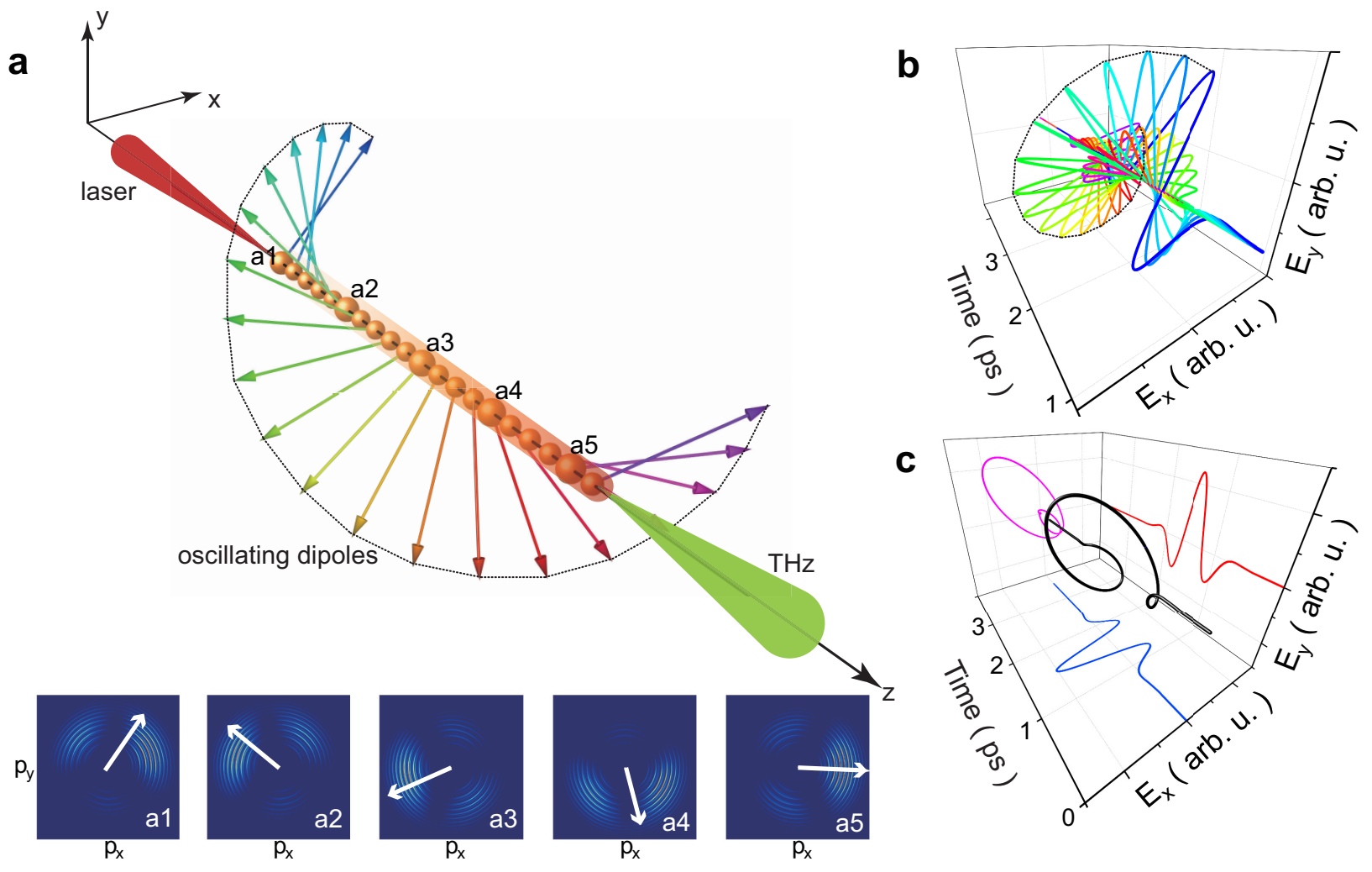

FIG. 2. The diagrams interpreting polarization of far-field terahertz radiation from a filament. (a) A schematic of a filament consists of a linear array of oscillating dipoles located along the filament (z-axis). The oscillating direction of each dipole is marked as a coloured arrow in the xy-plane. The insets are some snapshots of momentum distributions of ionized electrons in the $\left(p_{x}, p_{y}\right)$ plane obtained from numerical simulation for a short plasma driven by a two-colour pump laser with $\varphi_{d}=0.85 \pi, 1.26 \pi, 1.67 \pi, 2.08 \pi, 2.49 \pi$ from (a1) to (a5). These dipole oscillations (marked as a white arrow in each inset) are equivalent to those distributed along a long filament. (b) Far-field terahertz waves emitted from an array of dipoles located along the filament. Each waveform corresponds to a terahertz electric field $\left[E_{x}(t), E_{y}(t)\right]$ emitted from one dipole of the filament. (c) The polarization of the far-field terahertz pulse emitted from a long filament obtained by coherent superposition of linearly polarized terahertz fields from each dipole located along the filament shown in (b) in two orthogonal directions.

that the azimuthal angle $\theta^{\mathrm{THz}}$ can be manipulated by $\varphi_{d}$, while its ellipticity $\varepsilon^{\mathrm{THz}}$ can be controlled by the length of the filament.

The above observation can be explained by the oscillating dipole model based upon field ionization of atoms by the two-colour laser having different polarizations. When the two-colour laser pulse propagates through air, ionized electrons in the plasma zone acquire a drift velocity ${ }^{27,28}$, and the displacement of the positive and negative charges in the plasma zone forms the dipole. When the formed plasma filament is short, the dipole oscillation appears as a point source, which emits a terahertz pulse with linear polarization parallel to the direction of the dipole oscillation $^{29}$. However, when the plasma filament is extended to more than centimetre-long, the polarization of the far-field terahertz pulses is affected by the following two factors. First, there is a continuous change in the relative phase between the $\mathrm{FW}$ and its $\mathrm{SH}$ along a long filament due to their velocity mismatch, which results in a linear array of dipoles having variable amplitudes and oscillating directions along the filament (as coloured arrows in Fig. 2a). The dipole can be calculated using the strong field approximation (SFA) algorithm (See Methods for calculation of oscillating dipoles). Second, the velocity mismatch between the two-colour laser and the terahertz pulses results in an asynchronization in the wave fronts of the terahertz waves from different dipoles distributed along the filament. As shown in Fig. 2b, the terahertz pulse (blue waveform) emitted from the dipole located at the beginning of the filament (a1) propagates at the leading edge of all terahertz pulses from the filament, while that (purple waveform) from the end of the filament (a5) is at the tailing edge. After taking these two effects into account, polarization of far-field terahertz radiation from a filament can be obtained by coherently superposing all terahertz pulses from a linear array of dipoles along the filament (Fig. 2c) (See Methods for terahertz polarization using linear-dipole array model), and its chirality is determined by the rotation direction of the dipoles along the filament. The experimental observations shown in Fig. 1b and Fig. 1d are well reproduced theoretically in Fig. 1c and Fig. 1e for a short 
filament and for a long filament, respectively.

Domain of polarization variation for terahertz pulses with $\varphi_{d}$. When varying $\varphi_{d}$ from 0 to $2 \pi$, the tips of the maximum electric field vector (MEV) in each polarization trajectory form a global ellipse, which can be described by its ellipticity and azimuthal angle $(\Sigma, \Theta)$ as shown by the dotted curves in Fig. 1c and Fig. 1e for different filament lengths (See Methods for trail described by tips of MEV). This ellipse is termed as domain of polarization variation (DPV) for terahertz waves and the polarization transformation of the terahertz waves with $\varphi_{d}$ is localized inside DPV. To clarify the important role of DPV in manipulating the polarization of the emitted terahertz waves, we introduce two variables. First, the surface area swept out by a MEV is denoted as $\Delta S_{\mathrm{SW}}$ (shaded sectors in an ellipse in Fig. 3a) when $\varphi_{d}$ is changed by $\Delta \varphi_{d}$. The case for $\Delta S_{\mathrm{SW}}>0$ is defined as the anticlockwise rotation of MEV (i.e. the azimuthal angle of the polarization trajectory) when $\Delta \varphi_{d}>0$, and vice versa. When $\Delta S_{\mathrm{SW}}=0$, it means that the MEV stays still regardless of any change in $\varphi_{d}$. Second, for a given $\varphi_{d}$ and a corresponding elliptically polarized terahertz pulse, the area enclosed by the field of this terahertz pulse is expressed as $S_{\mathrm{EN}}$ (shaded areas in Fig. 3b). The case for $S_{\mathrm{EN}}>0$ is for terahertz polarization trajectory with a left-hand chirality, and vice versa. $S_{\mathrm{EN}}=0$ represents a linearly polarized terahertz signal.

It is found both theoretically and experimentally that there are two conservation relations:

$$
\begin{gathered}
\frac{\partial S_{\mathrm{SW}}}{\partial \varphi_{d}}=\text { constant } \\
\frac{\partial S_{\mathrm{EN}}}{\partial \varphi_{d}}=0 .
\end{gathered}
$$

Equation (1) means that a MEV sweeps out equal areas during equal intervals of $\varphi_{d}$. As schematically illustrated in Fig. 3a, the three shaded sectors are same for three equal intervals. This conservation relation suggests that the MEV rotates with $\varphi_{d}$ at a steady rate and the MEV will keep a constant magnitude when $\varphi_{d}$ changes provided that DPV is a circle. Equation (2) means that the elliptical area enclosed by the terahertz electric field keeps constant when $\varphi_{d}$ varies, as shown by the shaded ellipse $S_{\mathrm{EN} 1}$ and $S_{\mathrm{EN} 2}$ in Fig. $3 \mathrm{~b}\left(S_{\mathrm{EN} 1}=S_{\mathrm{EN} 2}\right)$. Generally for a DPV ellipse with a certain ellipticity $\Sigma<1$, not only the azimuthal angle but also the ellipticity of an emitted terahertz pulse changes with $\varphi_{d}$. Thus, a circular DPV or $\Sigma=1$ is required to keep the ellipticity of a polarization ellipse constant when varying $\varphi_{d}$. Therefore, by designing a circular DPV for terahertz polarization, we can make the azimuthal angle of an emitted terahertz polarization ellipse to rotate with $\varphi_{d}$ at a steady rate while freezing its ellipticity and peak field magnitude for any $\varphi_{d}$.

Manipulation of terahertz polarization. It is a prerequisite to acquire a desired circular DPV for tera-
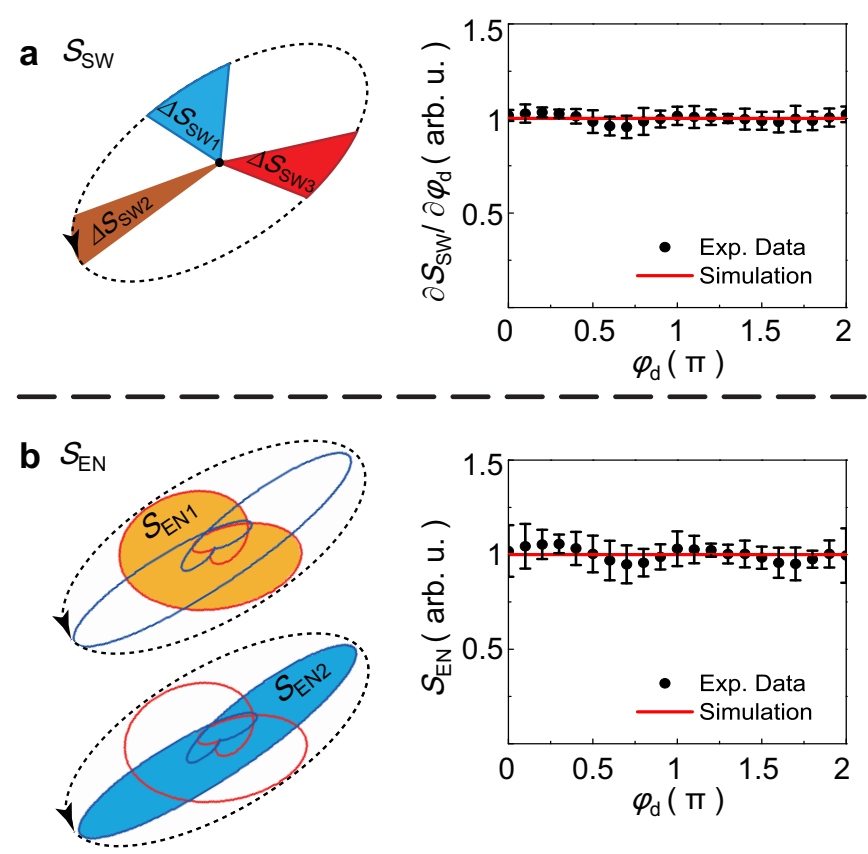

FIG. 3. Conservation variables related to terahertz polarization. (a) Schematic diagram and experimental data of the surface area swept out by a $\operatorname{MEV}\left(\Delta S_{\mathrm{SW}}\right)$ while $\varphi_{d}$ changed by an identical interval $\Delta \varphi_{d}$, where $\Delta S_{\mathrm{SW} 1}=$ $\Delta S_{\mathrm{SW} 2}=\Delta S_{\mathrm{SW} 3}$ or $\partial S_{\mathrm{SW}} / \partial \varphi_{d}=$ constant. (b) Schematic diagram and experimental data of the area $\left(S_{\mathrm{EN}}\right)$ enclosed by an elliptically polarized terahertz pulse $\left[E_{x}(t), E_{y}(t)\right]$ obtained with different $\varphi_{d}$, i.e., $S_{\mathrm{EN}}\left(\varphi_{d 1}\right)=S_{\mathrm{EN}}\left(\varphi_{d 2}\right)$. Black dotted curves in (a) and (b) indicate DPV for terahertz polarization formed when $\varphi_{d}$ changes by $2 \pi$. Each dot represents an averaged value of five measurements. Error bars in (a) and (b) represent standard deviation of five measurements.

hertz polarization control. Our theoretical model suggests that this can be realized by manipulating the polarization of the two-colour pump laser (See Methods for calculation of DPV). Concerning our experimental setup, the polarization of the SH pulse is fixed $\left(\varepsilon^{2 \omega}=\right.$ $0, \theta^{2 \omega}=\pi / 2$ ) while the polarization of the $\mathrm{FW}$ pulse can be controlled by tuning the DWP and QWP located after the IPC simultaneously. Thus, the ellipticity $\Sigma$ and azimuthal angle $\Theta$ of the DPV versus the polarization of the $\mathrm{FW}$ pulse $\left(\varepsilon^{\omega}, \theta^{\omega}\right)$ can be calculated by our model (Fig. 4a and Fig. 4b). Several examples for achieving different DPV are demonstrated in Fig. 4c by overlapping the contour maps for $\Sigma$ (solid lines for some specific values, derived from Fig. 4a) and $\Theta$ (dashed lines for some specific values, derived from Fig. 4b). Each intersection of a solid line and a dashed line illustrates a specified DPV with certain $(\Sigma, \Theta)$. As discussed above, effective control of the polarization of an emitted terahertz wave by $\varphi_{d}$ requires a circular DPV, i.e., $\Sigma= \pm 1$ and $\Theta$ being arbitrary. This special case is revealed by the intersections of several dashed lines inside the pink curve $(\Sigma=+1$, markedastriangle $)$ and purple curve $(\Sigma=-1$, markedasstar) in Fig. 4c. Here, $\Sigma=+1$ (or 

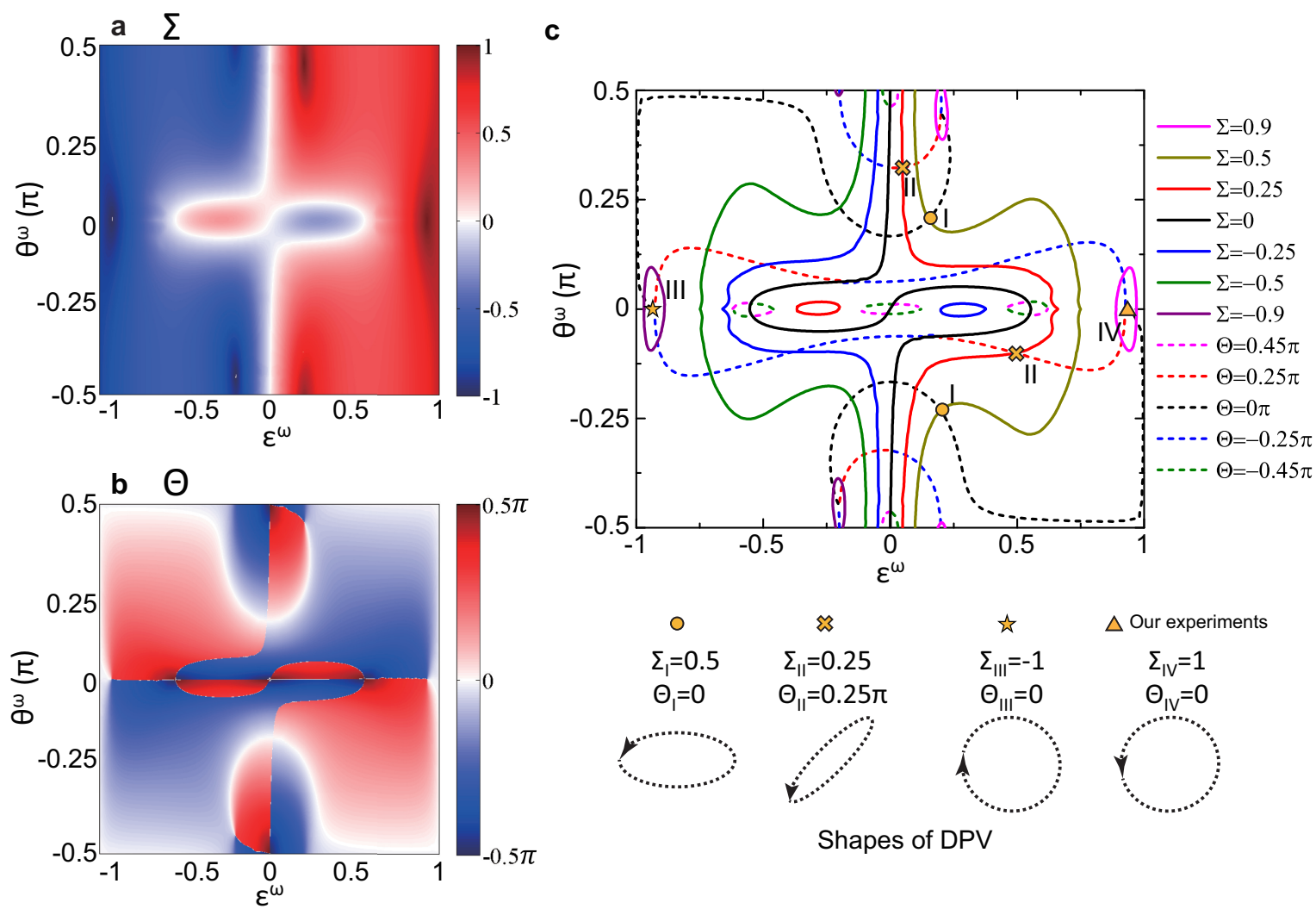

FIG. 4. Manipulation of DPV for terahertz waves by changing polarization of FW laser. Diagrams of ellipticity $\Sigma$ (a) and azimuthal angle $\Theta$ (b) of a trail described by tips of MEV as a function of the polarization of the FW pulse $\left(\varepsilon^{\omega}, \theta^{\omega}\right)$. (c) Diagram of overlapped contour maps of for certain $\Sigma$ (solid lines) and $\Theta$ (dashed lines), where four cases to realize certain DPV conditions for $(\Sigma, \Theta)=(0.5,0),(0.25,0.25 \pi),(-1,0),(1,0)$ are marked in the space $\left(\varepsilon^{\omega}, \theta^{\omega}\right)$. The triangle indicates the FW polarization condition for our experiment with a nearly circular trail described by tips of MEV. The circle, cross and star correspond to simulated DPVs with different ellipticities, azimuthal angles or chiralities.

$\Sigma=-1)$ means a circular MEV trail rotates anticlockwise (or clockwise) with $\varphi_{d}$. As mentioned above, the chirality of an emitted terahertz pulse follows the rotation direction of oscillating dipoles along the filament which is equivalent to the rotation direction of MEV when increasing $\varphi_{d}$, therefore the sign of $\Sigma$ is the key to control the chirality of the terahertz polarization.

Based on above discussions, parameters for the FW polarization in the experiment are designed as case IV $\left(\varepsilon^{\omega}=0.95, \theta^{\omega}=0\right)$ marked with a triangle in Fig. 4c, which is very close to the case for a desired circular DPV $(\Sigma=+1)$. Concerning this design, the terahertz polarization as a function of $\varphi_{d}$ varying from 0 to $2 \pi$ is simulated for some typical filament lengths (Fig. 5a1 - Fig. 5a5) (See Methods for calculation of DPV and control of terahertz polarization). The chirality of the terahertz trajectory is left-handed, following the trail described by tips of MEV with anticlockwise rotation $(\Sigma=+1)$. This polarization changes with $\varphi_{d}$ with a period of $2 \pi$. The simulation result verifies that the terahertz polarization can be effectively manipulated in case of a circular DPV. First, the azimuthal angle of the terahertz polarization $\left(\theta^{\mathrm{THz}}\right)$ can be rotated at a steady speed with $\varphi_{d}$ (i.e.,
$\theta^{\mathrm{THz}}=\varphi_{d}+$ constant, derived from Eq. (15) in Methods), while keeping its ellipticity and chirality unchanged. This observation was reproduced exactly in experiments with filament length of $4 \mathrm{~mm}$ and $23 \mathrm{~mm}$ (Fig. 5b). Meanwhile, the ellipticity of the terahertz polarization can be controlled by modifying the length of a filament. When the plasma channel changes from a point source to a filament with length of $18 \mathrm{~mm}$, the terahertz polarization varies gradually from a linear polarization $\left(\varepsilon^{\mathrm{THz}}=0\right.$, Fig. 5a1) to an elliptical one $\left(0<\varepsilon^{\mathrm{THz}}<1\right.$, Fig. 5a2 and Fig. 5a3) and then a circular one $\left(\varepsilon^{\mathrm{THz}} \sim 1\right.$, Fig. $5 \mathrm{a} 4$ ). When the filament grows even longer, the terahertz electric fields emitted from the beginning and tailing of the filament start to be out of phase with each other, resulting in a terahertz polarization evolving from circular $\left(\varepsilon^{\mathrm{THz}} \sim 1\right.$, Fig. 5a4) to elliptical $\left(0<\varepsilon^{\mathrm{THz}}<1\right.$, Fig. 5a5). Thus, the dependence of the ellipticity of terahertz polarization on the filament length can be clearly illustrated (Fig. 5c). The ellipticities found in the experiment for four different filament lengths agree well with this theoretical prediction. The theoretical and experimental results shown above demonstrate clearly that arbitrary terahertz polarization can be realized by controlling the 
a
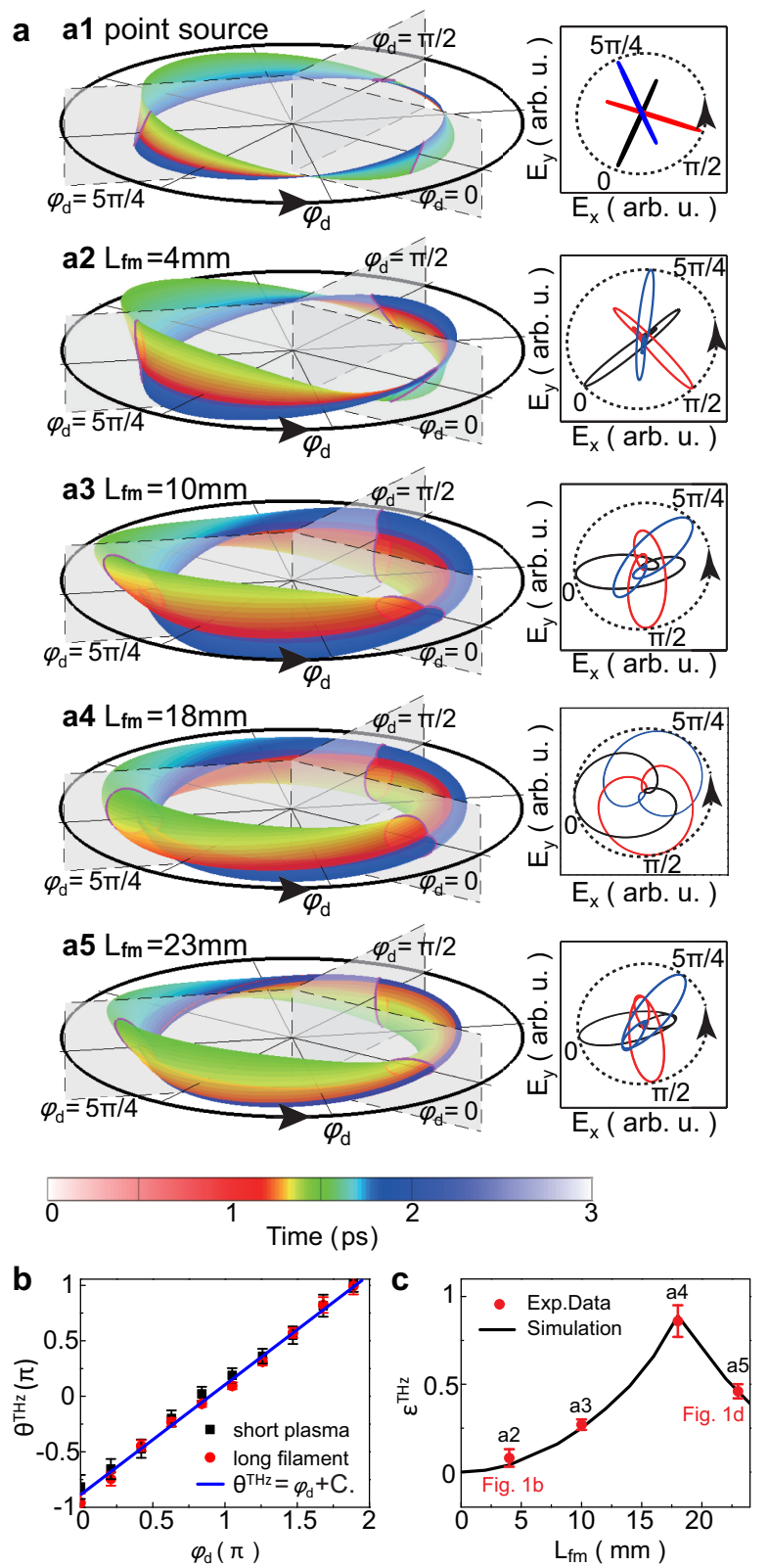

FIG. 5. Control of terahertz polarization with arbitrary azimuthal angle and ellipticity. (a) Evolution of terahertz polarization with $\varphi_{d}$ varying from 0 to $2 \pi$ in polar coordinates, emitting from a filament with different lengths $L_{\mathrm{fm}}$ ranging from a plasma point source to $23 \mathrm{~mm}$. The polar radian represents the value of $\varphi_{d}$. Three cross sections in (a1)-(a5) represent terahertz polarization trajectories obtained at $\varphi_{d}=0, \pi / 2,5 \pi / 4$ as shown in the corresponding insets on the right. (b) Dependence of the azimuthal angle of terahertz pulses on $\varphi_{d}$. The black rectangles and red dots are the experimental data from Fig. $1 \mathrm{~b}$ and $1 \mathrm{~d}$, and the blue line is the linear fit. C: constant. (c) Dependence of the ellipticity of the terahertz pulses on the filament length (black line) with the experimental data (red dots). initial relative phase between the FW and its $\mathrm{SH}$ and the filament length, as long as the DPV is circular.

\section{SUMMARY}

In conclusion, we have demonstrated theoretically and experimentally the generation of terahertz pulses with arbitrary polarization from a two-colour laser filament at far-field. As a result, we have the following recipe for achieving a broadband terahertz wave with any desired polarization: first, create a laser plasma filament using a two-colour laser with a circularly polarized $\mathrm{FW}$ and a linearly polarized SH; second, manage the effective filament length with suitable input laser energy to acquire a desired ellipticity for the terahertz polarization; third, adjust $\varphi_{d}$ to obtain a desired azimuthal angle for a terahertz pulse with elliptical polarization; finally, change the polarization of the $\mathrm{FW}$ to switch the chirality of the terahertz polarization. This work offers a practical technique on the flexible control of polarization property of broadband terahertz pulses, which can find wide applications in materials analysis, structural biology, remote sensing and communication in terahertz ranges.

\section{METHODS}

Measurement of terahertz polarization. An elliptically polarized wave can be decomposed into two mutually orthogonal field components with a fixed phase difference between them. By measuring the terahertz electric fields in two orthogonal directions, it is feasible to recover the elliptical polarization trajectory. In our experiment, the terahertz electric fields in two orthogonal directions can be measured by the electro-optical sampling method. For a given $\varphi_{d}$, we define the terahertz electric field obtained along x-axis as $E_{x}^{\mathrm{THz}}$ and the terahertz electric field along y-axis as $E_{y}^{\mathrm{THz}}$. Thus, a terahertz polarization trajectory for the given $\varphi_{d}$ can be observed on the $E_{x}-E_{y}$ plane (Fig. 1b and Fig. 1d). For such nearly single-cycle terahertz pulses, the measured elliptical polarization trajectories are not perfect ellipses due to their broad bandwidths.

Calculation of oscillating dipoles. The states of oscillating dipoles, caused by the final drift velocity of electrons driven by the two-colour laser field, can be acquired by simulating the momentum distributions of the ionized electrons using the Strong-Field-Approximation (SFA) algorithm. According to SFA, the quantum transition amplitude for obtaining a photoelectron with momentum $\boldsymbol{p}$ is described as:

$$
M(\boldsymbol{p})=-i \int_{t_{i}}^{t} d t^{\prime}\left\langle\Psi_{\boldsymbol{p}}^{(V)}\left(t^{\prime}\right)\left|\Omega_{\boldsymbol{E}}\left(t^{\prime}\right)\right| \Psi_{0}\left(t^{\prime}\right)\right\rangle
$$

where $\Psi_{p}^{(V)}(t)$ is the Volkov state and

$$
\left|\Psi_{\boldsymbol{p}}^{(V)}(t)\right\rangle=\exp \left[-i S_{\boldsymbol{p}}(t)\right] \cdot|\boldsymbol{p}+\boldsymbol{A}(t)\rangle
$$


with the Volkov phase

$$
S_{\boldsymbol{p}}(t)=\frac{1}{2} \int_{t_{i}}^{t} d t^{\prime}\left[\boldsymbol{p}+\boldsymbol{A}\left(t^{\prime}\right)\right]^{2} .
$$

Here $\boldsymbol{A}(t)$ is the laser vector potential and the electric field $\boldsymbol{E}(t)=-\partial \boldsymbol{A}(t) / \partial t . \quad \Psi_{0}(t)$ is the initial state and $\Omega_{\boldsymbol{E}}(t)=\boldsymbol{E}(t) \cdot \boldsymbol{r}$ represents the laser?electron interaction. For simplicity, we have used the ground state of hydrogen as the initial state in our SFA calculations.

The laser electric field is expressed as

$$
\begin{aligned}
\boldsymbol{E}(t) & =\frac{\left|E^{\omega}\right| e^{i \omega t}}{\sqrt{1+\left(\varepsilon^{\omega}\right)^{2}}}\left[\begin{array}{cc}
\cos \theta^{\omega} & -\sin \theta^{\omega} \\
\sin \theta^{\omega} & \cos \theta^{\omega}
\end{array}\right]\left[\begin{array}{c}
i \\
\varepsilon^{\omega}
\end{array}\right] \\
& +\left|E^{2 \omega}\right| e^{i 2 \omega t}\left[\begin{array}{c}
0 \\
e^{i \varphi_{d}}
\end{array}\right]
\end{aligned}
$$

where the first term on the right is the electric field of the FW with amplitude $\left|E^{\omega}\right|$, ellipticity $\varepsilon^{\omega}$ and azimuthal angle $\theta^{\omega}$. The second term represents the electric field of the SH with amplitude $\left|E^{2 \omega}\right|$ and a relative phase regarding the $\mathrm{FW} \varphi_{d}$. The absolute value of $\varepsilon^{\omega}$ varies between 0 to 1 , representing a linear polarization to a circular polarization. $\varepsilon^{\omega}>0$ means a left-handed polarization trajectory while $\varepsilon^{\omega}<0$ means a right-handed one. In our experiment, the intensities at the focus were about $10^{14} \mathrm{~W} / \mathrm{cm}^{2}$ for the $\mathrm{FW}$ and $10 \%$ of the $\mathrm{FW}$ for the $\mathrm{SH}$, respectively.

In the numerical simulation, the vector $\boldsymbol{p}$ is given in a two-dimension matrix. So the momentum distribution of ionized electrons in a plane perpendicular to laser propagation can be calculated by $M^{*}(\boldsymbol{p}) M(\boldsymbol{p})$, as shown in Fig. 2a1-2a5. And the corresponding final oscillating dipole can be derived as

$$
\boldsymbol{P}=\iint_{\boldsymbol{p}} \boldsymbol{p} M^{*}(\boldsymbol{p}) M(\boldsymbol{p}) d \boldsymbol{p} .
$$

Terahertz polarization calculation with lineardipole array model. The key idea of the linear-dipole array (LDA) model is that a plasma filament is considered as an aligned array of point sources radiating terahertz pulses. Thus, the far-field terahertz waveforms can be regarded as coherent superposition of terahertz pulses emitted from a linear-dipole array along the filament. When each point source in the filament is regarded as an oscillating dipole, its radiation in the emission angle $\Omega$ can be expressed as:

$$
\begin{aligned}
d \boldsymbol{E}_{\text {dipole }}^{\mathrm{THz}}\left(\omega^{\mathrm{THz}}, z, t\right) & =\frac{-\left(\omega^{\mathrm{THz}}\right)^{2} \cos \Omega}{4 \pi \epsilon_{0} c^{3} R(z, \Omega)} d \boldsymbol{P}\left(\varphi_{d}, z\right) \\
& \times \exp \left(-j \omega^{\mathrm{THz}} t\right) \exp [j \Phi(z, \Omega)](8)
\end{aligned}
$$

where $R(z, \Omega)$ is the propagation length of the terahertz wave from its original position $z, \Phi(z, \Omega)$ is the wave phase of the radiation, $d \boldsymbol{P}\left(\varphi_{d}, z\right)$ is the dipole moment calculated using SFA algorithm.

Here we assume that terahertz radiation emitted by the filament at the propagation coordinate $z$ does not affect the emission at $z+d z$. Thus,

$$
\boldsymbol{E}^{\mathrm{THz}}\left(\omega^{\mathrm{THz}}, t\right)=\int_{\text {filament }} d \boldsymbol{E}_{\text {dipole }}^{\mathrm{THz}}\left(\omega^{\mathrm{THz}}, z, t\right)
$$

where the integration range is the filament length. Consequently, the polarization of the terahertz radiation can be described by Eq. (9). If we let the normalized wave function $W(t, z)=\exp \left(-j \omega^{\mathrm{THz}} t\right) \exp [j \Phi(z, \Omega)]$ and $\boldsymbol{A}^{\mathrm{THz}}\left(\varphi_{d}, z\right)=\frac{-\left(\omega^{\mathrm{THz}}\right)^{2} \cos \Omega}{4 \pi \epsilon_{0} c^{3} R(z, \Omega)} d \boldsymbol{P}\left(\varphi_{d}, z\right)$, we will obtain

$$
\boldsymbol{E}^{\mathrm{THz}}\left(\varphi_{d}, t\right)=\int_{\text {filament }}\left[\begin{array}{c}
A_{x}^{\mathrm{THz}}\left(\varphi_{d}, z\right) \\
A_{y}^{\mathrm{THz}}\left(\varphi_{d}, z\right)
\end{array}\right] \cdot W(t, z) d z
$$

Trail described by tips of MEV of terahertz pulses. The carrier-envelope phase of a terahertz pulse emitted from a plasma point source (or a short filament) features with phase-locked behavior as $\varphi_{d}$ changes. In this case, the terahertz waveform can be written as

$$
E_{\text {short }}^{\mathrm{THz}}\left(\varphi_{d}, t\right)=\left[\begin{array}{l}
A_{x}^{\mathrm{THz}}\left(\varphi_{d}\right) \\
A_{y}^{\mathrm{THz}}\left(\varphi_{d}\right)
\end{array}\right] \cdot W(t)
$$

where $W(t)$ is normalized waveform function which is independent of $\varphi_{d}$. Here $A_{x}^{\mathrm{THz}}\left(\varphi_{d}\right)$ and $A_{y}^{\mathrm{THz}}\left(\varphi_{d}\right)$ are amplitudes of terahertz waves at $\mathrm{x}$-direction and $\mathrm{y}$-direction, respectively. Both of them are derived from final drift velocities of ionized electrons induced by two-colour laser field using the SFA method (see Methods section 'Calculation of oscillating dipoles'). Simulation results show that terahertz amplitudes change sinusoidally with $\varphi_{d}$, i.e.,

$$
\left[\begin{array}{l}
A_{x}^{\mathrm{THz}}\left(\varphi_{d}\right) \\
A_{y}^{\mathrm{THz}}\left(\varphi_{d}\right)
\end{array}\right]=\left[\begin{array}{l}
A_{x 0}^{\mathrm{THz}} \cos \left(\varphi_{d}-\Phi_{x}\right) \\
A_{y 0}^{\mathrm{THz}} \cos \left(\varphi_{d}-\Phi_{y}\right)
\end{array}\right]
$$

where $A_{x 0}^{\mathrm{THz}}$ and $A_{y 0}^{\mathrm{THz}}$ are repsectively the maximum terahertz amplitudes along the $\mathrm{x}$ direction and $\mathrm{y}$-direction when $\varphi_{d}$ changes from 0 to $2 \pi$. $\Phi_{x}$ and $\Phi_{y}$ are the corresponding values for $\varphi_{d}$ when terahertz amplitudes at $\mathrm{x}$-direction and $\mathrm{y}$-direction reach their maximums. In geometry, Eq. (12) is a parametric equation of an ellipse, which indicates that the polarization of the terahertz radiation rotates with the change of $\varphi_{d}$ and the trail described by tips of MEV in each terahertz polarization follows an ellipse.

As a plasma point source extends to a long filament, the terahertz radiation can be calculated using LDA model (See above), i.e., Eq. (10). The trail described by tips of MEV for each point source along the filament satisfies the following parametric equation

$$
\left[\begin{array}{l}
A_{x}^{\mathrm{THz}}\left(\varphi_{d}, z\right) \\
A_{y}^{\mathrm{THz}}\left(\varphi_{d}, z\right)
\end{array}\right]=\left[\begin{array}{l}
A_{x 0}^{\mathrm{THz}} \cos \left[\varphi_{d}-\Phi_{x}+\phi(z)\right] \\
A_{y 0}^{\mathrm{THz}} \cos \left[\varphi_{d}-\Phi_{y}+\phi(z)\right]
\end{array}\right]
$$

where $\phi(z)$ is induced by the group velocity mismatch between two-colour laser components. Obviously, this equation describes a trajectory of an ellipse which features with the same ellipticity and azimuthal angle as 
that described by Eq. (13). This result leads to a conclusion, using mathematical induction, that the trail described by tips of MEV will not change with the length of a filament and its shape is decided by parameter equation Eq. (12). Our simulation using LDA model shows that the polarization of the two-colour laser field can be the key to control the parameters $A_{x 0}, A_{y 0}, \Phi_{x}, \Phi_{y}$ in Eq. (12), and consequently shaping the trail described by tips of MEV.

Calculation of DPV and control of terahertz polarization. Using SFA algorithm and LDA model, we can calculate the parameters $A_{x 0}, A_{y 0}, \Phi_{x}, \Phi_{y}$ with a given two-colour laser field as Eq. (6). Substituting these parameters into Eq. (12), we can obtain the ellipticity $\Sigma$ and the azimuthal angle $\Theta$ of the trail described by tips of $\mathrm{MEV}$ by solving the following equation.

$$
\left[\begin{array}{c}
A_{x 0}^{\mathrm{THz}} \cos \left(\varphi_{d}-\Phi_{x}\right) \\
A_{y 0}^{\mathrm{THz}} \cos \left(\varphi_{d}-\Phi_{y}\right)
\end{array}\right]=\operatorname{Re}\left(e^{i \varphi_{d}}\left[\begin{array}{cc}
\cos \Theta & -\sin \Theta \\
\sin \Theta & \cos \Theta
\end{array}\right]\left[\begin{array}{c}
i \\
\Sigma
\end{array}\right]\right)
$$

In addition, we can calculate the dependence of the azimuthal angle of terahertz polarization on $\varphi_{d}$ as

$$
\theta^{\mathrm{THz}}=\tan ^{-1} \frac{-\Sigma \sin \Theta \cos \varphi_{d}-\cos \Theta \sin \varphi_{d}}{\Sigma \cos \Theta \cos \varphi_{d}-\sin \Theta \sin \varphi_{d}}+C
$$

As long as DPV is a shape of circle, i.e., $\Sigma= \pm 1$, Eq. (15) becomes $\theta^{\mathrm{THz}}= \pm \varphi_{d}+C$, where $C$ is an arbitrary constant.

${ }^{1}$ Y. Yang, K. Kelley, E. Sachet, S. Campione, T. S. Luk, J.-P. Maria, M. B. Sinclair, and I. Brener, Nat. Photon. 64, (2017).

${ }^{2}$ M. Sato, T. Higuchi, N. Kanda, K. Konishi, K. Yoshioka, T. Suzuki, K. Misawa, and M. Kuwata-Gonokami, Nat. Photon. 7, 724 (2013).

${ }^{3}$ T. L. Cocker, V. Jelic, M. Gupta, S. J. Molesky, J. A. Burgess, G. De Los Reyes, L. V. Titova, Y. Y. Tsui, M. R. Freeman, and F. A. Hegmann, Nat. Photon. 7, 620 (2013).

${ }^{4}$ J. Zhu, Z. Ma, W. Sun, F. Ding, Q. He, L. Zhou, and Y. Ma, Appl. Phys. Lett. 105, 021102 (2014),

${ }^{5}$ B.-X. Wang, L.-L. Wang, G.-Z. Wang, W.-Q. Huang, X.-F. Li, and X. Zhai, Appl. Phys. A 115, 1187 (2014).

${ }^{6}$ X. Zang, C. Shi, L. Chen, B. Cai, Y. Zhu, and S. Zhuang, Sci. Rep. 5, 8901 (2015).

${ }^{7}$ R. Singh, E. Plum, C. Menzel, C. Rockstuhl, A. K. Azad, R. A. Cheville, F. Lederer, W. Zhang, and N. I. Zheludev, Phys. Rev. B 80, 153104 (2009).

${ }^{8}$ S. Baierl, M. Hohenleutner, T. Kampfrath, A. K. Zvezdin, A. V. Kimel, R. Huber, and R. V. Mikhaylovskiy, Nat. Photon. 10, 715 (2016).

${ }^{9}$ S. Katletz, M. Pfleger, H. Pühringer, M. Mikulics, N. Vieweg, O. Peters, B. Scherger, M. Scheller, M. Koch, and K. Wiesauer, Opt. Express 20, 23025 (2012).

${ }^{10}$ H. Hoshina, Y. Morisawa, H. Sato, H. Minamide, I. Noda, Y. Ozaki, and C. Otani, Phys. Chem. Chem. Phys. 13, 9173 (2011).

${ }^{11}$ M. Tonouchi, Nat. Photon. 1, 97 (2007).
${ }^{12}$ N. Amer, W. C. Hurlbut, B. J. Norton, Y.-S. Lee, and T. B. Norris, Appl. Phys. Lett. 87, 221111 (2005), .

${ }^{13}$ A. Houard, Y. Liu, B. Prade, V. T. Tikhonchuk, and A. Mysyrowicz, Phys. Rev. Lett. 100, 255006 (2008).

${ }^{14}$ Y. Chen, T.-j. Wang, C. Marceau, F. Théberge, M. Châteauneuf, J. Dubois, O. Kosareva, and S. L. Chin, Appl. Phys. Lett. 95, 101101 (2009).

${ }^{15}$ X. Lu and X.-C. Zhang, Phys. Rev. Lett. 108, 123903 (2012).

${ }^{16}$ J. Dai, N. Karpowicz, and X.-C. Zhang, Phys. Rev. Lett. 103, 023001 (2009).

${ }^{17}$ H. Wen and A. M. Lindenberg, Phys. Rev. Lett. 103, 023902 (2009).

${ }^{18}$ Y. S. You, T. I. Oh, and K.-Y. Kim, Opt. Lett. 38, 1034 (2013).

${ }^{19}$ J.-M. Manceau, M. Massaouti, and S. Tzortzakis, Opt. Express 18, 18894 (2010).

${ }^{20}$ H. Wang, N. Li, Y. Bai, P. Liu, Z. Wang, and C. Liu, Opt. Express 25, 30987 (2017).

${ }^{21}$ C. Meng, W. Chen, X. Wang, Z. Lü, Y. Huang, J. Liu, D. Zhang, Z. Zhao, and J. Yuan, Appl. Phys. Lett. 109, 131105 (2016).

${ }^{22}$ L. Zhang, W. Wang, T. Wu, R. Zhang, S. Zhang, C. Zhang, Y. Zhang, Z. Sheng, and X. Zhang, Phys. Rev. Lett. 119, 235001 (2017).

${ }^{23}$ Q. Wu and X.-C. Zhang, Appl. Phys. Lett. 71, 1285 (1997).

${ }^{24}$ P. C. M. Planken, H.-K. Nienhuys, H. J. Bakker, and T. Wenckebach, J. Opt. Soc. Am. B 18, 313 (2001).

${ }^{25}$ C. D'Amico, A. Houard, M. Franco, B. Prade, A. Mysyrowicz, A. Couairon, and V. T. Tikhonchuk, Phys. Rev. Lett. 98, 235002 (2007).

${ }^{26}$ Y. S. You, T. I. Oh, and K. Y. Kim, Phys. Rev. Lett. 109, $183902(2012)$.

${ }^{27}$ K. Y. Kim, J. H. Glownia, A. J. Taylor, and G. Rodriguez, Opt. Express 15, 4577 (2007).

${ }^{28}$ M. Chen, A. Pukhov, X.-Y. Peng, and O. Willi, Phys. Rev. E 78, 046406 (2008).

${ }^{29}$ Z. Zhang, Y. Chen, M. Chen, Z. Zhang, J. Yu, Z. Sheng, and J. Zhang, Phys. Rev. Lett. 117, 243901 (2016)

\section{ACKNOWLEDGEMENTS}

This work was supported by the National Basic Research Program of China (Grant No. 2014CB339801), the National Natural Science Foundation of China (Grant No. 11474202, 11655002, 11774228 and 11721091) and Science and Technology Commission of Shanghai Municipality (Grant No. 16DZ2260200). Z. S. acknowledges the support of a Leverhulme Trust Research Grant at University of Strathclyde.

\section{AUTHOR CONTRIBUTIONS}

Z.L.Z., Y.C. and Z.S. conceived the study and wrote the main manuscript. Z.L.Z. and Y.C. carried out the experiments and analyzed the data. Z.L.Z., S.C. and F.H. developed the theoretical model. F.H. and M.C. gave theoretical support. Z.Z., J.Y., L.C. and J.Z. gave experimental support. All authors discussed the results and commented on the manuscript. 